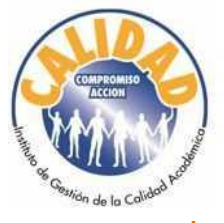

Vicerrectoría Académica

Instituto de Gestión de la Calidad Académica

Co-creando Excelencia $\square$

http://revistas.uned.ac.cr./index.php/revistacalidad

Carrea electrónica: revistacalidad国uned.ac.сг

\title{
Sistema de gestión de la calidad del proceso de extensión universitaria, una experiencia en la Universidad de La Habana.
}

\section{System quality managment for the university extension process, an experience of the Havana's University.}

Odette González-Aportela ${ }^{1}$

odette@rect.uh.cu

Universidad de La Habana, Cuba

https://orcid.org/0000-0002-8924-6976
Amado Batista-Mainegra ${ }^{2}$

amado@cepes.uh.cu

Universidad de La Habana, Cuba

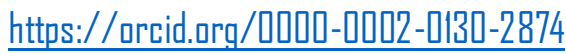

\section{Mercedes González Fernández-Larrea ${ }^{3}$ \\ mercedes.gonzalez@cepes.uh.cu \\ Universidad de La Habana, Cuba

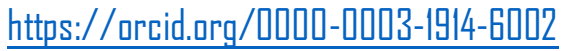

DOI: http://dx.doi.org/10.22458/caes.v11i2.3324

Volumen 11, Número 2

30 de noviembre de 2020

pp. $105-134$

Recibido: 27 de mayo del 2020

Aprobado: 28 de octubre del 2020

${ }^{1}$ Profesora Titular del Centro de Estudios para el Perfeccionamiento de la Educación Superior (CEPES) de la Universidad de La Habana. Doctora en Ciencias de la Educación, Master en Medio Ambiente y Desarrollo, Licenciada en Microbiología. Académica de Honor de la Academia Internacional de Ciencias, Tecnología, Educación y Humanidades (AICTEH). Directora de Extensión Universitaria de la Universidad de La Habana en el período 2011-2020.

2 Profesor Titular y Subdirector de Posgrado y Relaciones Internacionales del Centro de Estudios para el Perfeccionamiento de la Educación Superior (CEPES) de la Universidad de La Habana. Doctor en Ciencias de la Educación, Master en Ciencias de la Educación Superior, Licenciado en Microbiología. Académico de Honor de la Academia Internacional de Ciencias, Tecnología, Educación y Humanidades (AICTEH).

${ }^{3}$ Profesora Titular del Centro de Estudios para el Perfeccionamiento de la Educación Superior (CEPES) de la Universidad de La Habana. Doctora en Ciencias de la Educación. Máster en Promoción Cultural. Licenciada en Historia y Ciencias Sociales. Miembro del Tribunal Nacional de Grados Científicos de Ciencias de la Educación en Cuba.

Sistema de gestión de la calidad del procesa de extensión universitaria, una experiencia en la Universidad de La Habana.

Odette González-Apartela, Amada Batista-Mainegra, Mercedes González Fernández-Larrea

DOI: http://dx.dai.org/l0.22458/caes.villi2.3324

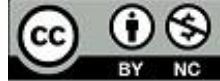

Artícula prategida par licencia Creative Cammans 


\title{
Resumen
}

La gestión de la calidad es una necesidad y oportunidad en las Instituciones de Educación Superior para responder con eficiencia y eficacia a las demandas de la sociedad; por ello los procesos universitarios deben actualizar sus mecanismos de gestión. El presente trabajo persigue como objetivo, desarrollar un sistema de gestión de la calidad del proceso de extensión universitaria en la Universidad de La Habana, para lo cual se empleó una investigación mixta concurrente. Se obtuvo un sistema de gestión de la calidad del proceso extensionista en la Universidad de La Habana, el cual consta de siete etapas (Fundamentación, Diseño estratégico, Diseño de procesos, Diseño evaluativo, Diseño integrado, Implementación y evaluación), que es contextualizado, flexible, democrático, entendible y operativo. Los usuarios manifestaron satisfacción y reconocimiento de la efectividad y utilidad de la propuesta una vez implementada.

Palabras clave: sistema, gestión de la calidad y extensión universitaria.

\begin{abstract}
Quality management is a necessity and opportunity in Higher Education Institutions, to respond efficiently and effectively to the demands of society; for this reason, university processes must update their management mechanisms. The objective of this work is to develop a quality management system for the university extension process at the University of Havana, for which a concurrent mixed investigation was used. A quality management system for the extension process was obtained at the University of Havana, which consists of seven stages (Foundations, Strategic Design, Process Design, Evaluative Design, Integrated Design, Implementation and Evaluation), which is contextualized, flexible, democratic, understandable and operational. Users expressed satisfaction and recognition of the effectiveness and usefulness of the proposal once it is implemented.
\end{abstract}

Keywords: system, quality management and university extension.

\section{Introducción}

Mejorar la calidad de la educación, en especial de la Educación Superior, es una prioridad y un desafío de todos los países del mundo. Los sistemas educacionales se encuentran sujetos a los constantes cambios en el orden político, económico, cultural, ambiental,

Sistema de gestión de la calidad del procesa de extensión universitaria, una experiencia en la Universidad de La Habana.

Odette Ganzález-Apartela, Amada Batista-Mainegra, Mercedes González Fernández-Larrea

DII: http://dx.dai.org/10.22458/caes.vlliz.3324

(c) (i) (8)

Artícula protegida par licencia Creative Commans 
tecnológico y social, donde constantemente los cambios tecnológicos y de innovación conllevan a las Instituciones de Educación Superior (IES) a trabajar, "en consonancia directa con los cambios y ser guía y líder de los avances de la ciencia y la tecnología" (González y Batista, 2019, p. 55) para responder así al desarrollo de la sociedad, contribuyendo al cumplimiento de su encargo social.

Tal y como se refrendó en la III Conferencia Regional de Educación Superior efectuada en Córdoba Argentina (CRES, 2018):

Se hace fundamental abordar el tema de la calidad, en un contexto de diversidad. Esto exige definir la calidad con la participación de los principales actores sociales, establecer indicadores apropiados a las características de los propósitos formativos de las instituciones (...). Se deben diseñar políticas y mecanismos que no se limiten a evaluar, sino también a promover la calidad (...). Las instituciones deben asumir el compromiso de avanzar de manera sostenida en su capacidad de respuesta a las necesidades de sus estudiantes y del entorno social en el que se desempeñan. (p. 11)

El desarrollo de la sociedad es una preocupación y ocupación de la educación, el cual debe responder de manera oportuna y eficaz a sus exigencias y asegurar un futuro promisorio a las nuevas generaciones. Ese es el compromiso y el desafío demandado a las Instituciones de Educación Superior (IES) por parte de las sociedades que las sustentan. Las IES participan en la solución de los problemas de la sociedad, en la construcción de un mundo mejor, un país más justo y responsable con la humanidad y en el desarrollo económico, político, cultural y social. En esta línea de pensamiento, Lemaitre et al. (2018) hacen referencia a que

es responsabilidad de las IES constituirse en espacio de construcción del conocimiento para dar respuesta a las necesidades de la sociedad (...) a su vez, la responsabilidad de cumplir con la misión de la institución con calidad, con

Sistema de gestión de la calidad del procesa de extensión universitaria, una experiencia en la Universidad de La Habana.

Odette Ganzález-Apartela, Amada Batista-Mainegra, Mercedes González Fernández-Larrea

DDI: http://dx.dai.org//0.22458/caes.vlli2.3324

(c)

Artícula prategida par licencia Creative Cammans 
pertinencia y con compromiso social. (...) Es necesario que las IES den continuidad a los procesos de análisis, revisión, reformulación y mejora continua, con una mirada crítica y en una prospectiva que se oriente a la consolidación de sus programas, su investigación, sus actividades de vinculación con el medio, para brindar una formación que dé respuesta a los problemas de la sociedad en la que se inserta la institución y contribuya con su desarrollo (p. 112).

Las IES cumplen su encargo social, a partir de la gestión de sus procesos, donde los procesos sustantivos de formación, investigación y extensión universitaria, contribuyen de manera sustancial al logro de la misión institucional. El proceso de extensión universitaria es aquel cuya esencia radica en la interacción dialéctica universidad sociedad, se identifica con el papel de promover la cultura ${ }^{4}$ en su más amplia acepción, en la comunidad intra y extrauniversitaria, la cual se articula con las otras dos funciones sustantivas y junto con estas contribuye al desarrollo sociocultural y de los valores (MES, 2010, p. 4).

La extensión universitaria se convierte, a partir de su práctica, en el elemento integrador y dinamizador que facilita la relación cultural continua y recíproca entre la IES y la sociedad, "garantiza un vínculo social más amplio y dinámico, que propicia la identificación, la comunicación y la actividad conjunta de los universitarios y la población en general, y estrecha su imprescindible unidad" (González y González, 2020, p. 6); es también un proceso de interacción humana, en tanto orienta sus propósitos no solo a contribuir al resto de las funciones universitarias y a la institución en sí misma, sino a colaborar con la formación integral de la comunidad intra y extrauniversitaria, así como favorece la

\footnotetext{
${ }^{4}$ Cultura, definida como "el conjunto de rasgos distintivos espirituales y materiales, intelectuales y afectivos que caracterizan una sociedad o un grupo social. Ella engloba, además de las artes y las letras, los modos de vida, los derechos fundamentales del ser humano, los sistemas de valores, las tradiciones y las creencias". (Maraña, 2010, p. 9).
}

Sis tema de gestión de la calidad del procesa de extensión universitaria, una experiencia en la Universidad de La Habana.

Odette González-Apartela, Amada Batista-Mainegra, Mercedes González Fernández-Larrea

DDI: http://dx.dai.org//0.22458/caes.vlli2.3324

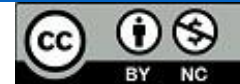

Artícula protegida par licencia Creative Cammans 
transformación de la sociedad mediante su propia participación en las perspectivas globales del entorno social.

El presente trabajo tiene como objetivo desarrollar un sistema de gestión de la calidad del proceso de extensión universitaria (SGC-EU) en la Universidad de La Habana (UH), por lo tanto, se muestra el procedimiento para diseñar, ejecutar y evaluar el SGC-EU, según el enfoque basado en procesos. Además, se exponen los principales conceptos asumidos, los elementos que componen el sistema y las principales valoraciones de los usuarios tras su implementación.

El diseño del SGC-EU se llevó a cabo desde el 2011, la etapa de Fundamentación comenzó en el primer semestre, seguida del Diseño estratégico (segundo semestre del año). Posteriormente, en el 2012, se trabajó en el Diseño de procesos y Diseño evaluativo y en el 2013, se concluyó el Diseño integrado. Se terminó la primera versión del SGC en el 2013, donde comienza su Implementación y evaluación, con la consecuente mejora continua del sistema, lo cual conllevó a cambios sustanciales para obtener el SGC-EU que se expone en el presente documento.

\section{Desarrollo}

Una forma de perfeccionar la gestión de las IES es a través de una gestión de calidad de sus procesos, en la Universidad de La Habana, se inicia este proceso en el 2011, con la identificación y definición de los procesos clave, estratégicos y de apoyo, para el cumplimiento de los objetivos de la organización, en los cuales se resaltan los procesos sustantivos de formación, investigación y extensión universitaria.

Son las instituciones culturales, y en especial las IES, las llamadas a ofrecer opciones de acción que le permitan al ser humano desenvolverse eficientemente en los nuevos

Sistema de gestión de la calidad del procesa de extensión universitaria, una experiencia en la Universidad de La Habana.

Odette González-Apartela, Amada Batista-Mainegra, Mercedes González Fernández-Larrea

DDI: http://dx.dai.org/l0.22458/caes.vlliz.3324

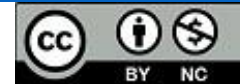

Artícula prategida par licencia Creative Cammans 
escenarios. Esta perspectiva resulta hoy un gran reto para los países latinoamericanos y del Caribe, respecto al escenario mundial, donde se vislumbra la necesidad de una gestión institucional de acuerdo con las exigencias inmediatas y futuras, tanto en lo organizacional como en lo político, cultural, técnico y conceptual. Es objeto de creciente atención de la dirección universitaria lograr una acción proactiva que garantice la interacción entre lo educativo y lo administrativo.

El acercamiento a la problemática de la gestión en la educación superior trae consigo una búsqueda permanente en su concepción, así como, una profundización respecto a los procesos sustantivos, ya que estos poseen formas propias de dirección, lo cual conlleva asegurar una capacidad superior de gestión, una mayor inserción del análisis de esta problemática de manera integrada a sus objetivos estratégicos y líneas de acción y una creciente implicación de la comunidad universitaria y los agentes sociales en el desarrollo de los procesos.

En los momentos actuales se exige el mejoramiento continuo de la gestión de los procesos que se desarrollan en las IES. La evaluación sistemática de la calidad de la gestión de estos procesos debe ser asumida como una vía para elevar de forma progresiva su efectividad en la gestión institucional en general. Para llevar a cabo una gestión de calidad es necesario adoptar un enfoque de gestión por procesos, y para ello la organización debe identificar todas y cada una de las actividades que ejecuta, ordenarlas y ubicarlas según su importancia para el cumplimiento de los objetivos de la organización para así desarrollar niveles superiores en la educación y en particular en las IES, específicamente en sus procesos.

La gestión por procesos lleva implícito un cambio cultural en la organización, cuya esencia es que cada persona que la integra entienda la relevancia de su trabajo y su participación

Sis tema de gestión de la calidad del procesa de extensión universitaria, una experiencia en la Universidad de La Habana.

Odette Ganzález-Apartela, Amada Batista-Mainegra, Mercedes González Fernández-Larrea

DDI: http://dx.dai.org/l0.22458/caes.vlliz.3324

(c) (i) (8)

Artícula prategida par licencia Creative Cammans 
dentro del proceso. El enfoque basado en procesos consiste en la identificación y gestión sistemática de los procesos desarrollados en la organización y en particular las interacciones entre tales procesos (ISO 9000:2000).

La ISO 9001 (2003) y la ISO 9001 (2015) pretenden fomentar la adopción del enfoque basado en procesos para gestionar una organización. La gestión por procesos, cuando se utiliza en el desarrollo, la implementación y la mejora de la eficacia de un Sistema de Gestión de la Calidad (SGC), concentra su atención en los siguientes puntos:

1. La comprensión y el cumplimiento de los requisitos de los clientes de cada proceso,

2. la necesidad de considerar y de planificar los procesos en términos que aporten valor, 3. el control, la medición y la obtención de resultados del desempeño y de la eficacia de los procesos,

4. la mejora continua de los procesos con base en mediciones objetivas.

La norma ISO 9001 (2001) establece las estructuras para llevar a cabo el enfoque basado en procesos. La trascendencia de este enfoque en esta norma es tan evidente que los propios contenidos se estructuran con este enfoque; esto permite a su vez concebir y entender los requisitos de la norma vinculados entre sí.

En el caso de las IES, en la literatura aparecen varias clasificaciones de procesos universitarios, en esta investigación se acepta la clasificación propuesta por Batista, Ortiz y González (2018), en la que se definen los procesos universitarios en procesos estratégicos, procesos clave o sustantivos (se hace referencia a los procesos fundamentales) y los procesos de apoyo. En consecuencia, se asumen como procesos sustantivos la formación, la investigación y la extensión universitaria.

Sis tema de gestión de la calidad del procesa de extensión universitaria, una experiencia en la Universidad de La Habana.

Odette Ganzález-Apartela, Amada Batista-Mainegra, Mercedes González Fernández-Larrea

DII: http://dx.dai.org/l0.22458/caes.vlli2.3324

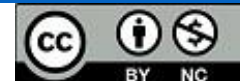

Artícula prategida par licencia Creative Cammans 
Tras un análisis exhaustivo acerca de la definición de proceso, se asume la propuesta por González (2016), la cual plantea que, un proceso es

el conjunto de actividades ${ }^{5}$ y tareas, definidas y específicas, que lo particularizan, y que permiten la transformación de las entradas de diferentes insumos en salidas, a partir de una gestión eficiente y eficaz, teniendo en cuenta los recursos humanos, de información, técnicos, financieros y materiales para contribuir al cumplimiento de la misión de la organización en interrelación con la sociedad. (p. 22)

A partir de esta definición se le otorga valor al proceso, este debe verse como exponencial en el tiempo, donde la participación de todos y cada uno de los involucrados afecta la calidad del resultado o producto final. En las organizaciones donde principalmente prestan servicios, como es el caso de la educación, el activo más importante que añade valor al producto final es el recurso humano: su calidad, compromiso, capacitación y experiencia. El propósito que ha de tener todo proceso es ofrecer al usuario un servicio que cubra sus necesidades y satisfaga sus expectativas, con el mayor grado de rendimiento en costo, servicio y calidad.

Sin embargo, no todos los procesos tienen la misma influencia para el cumplimiento de la misión o los objetivos generales de la institución, por lo que resulta conveniente clasificarlos teniendo en consideración su impacto en todos los ámbitos de desarrollo, según Galarza (2007). En los momentos actuales se exige el mejoramiento continuo de la gestión de los procesos que en las IES se desarrollan, entre los cuales se destacan aquellos catalogados como estratégicos, los fundamentales, que están vinculados con la formación de pre y posgrado, la investigación y la extensión universitaria, así como los de apoyo o soporte, entre otros; lo cual va aparejado de la evaluación sistemática de la calidad

\footnotetext{
${ }^{5}$ Actividad: acción repetitiva que se ejecuta mediante un procedimiento dado para contribuir a la obtención de los resultados de determinado proceso (Rodríguez et al., 2010).
}

Sistema de gestión de la calidad del procesa de extensión universitaria, una experiencia en la Universidad de La Habana.

Odette Ganzález-Apartela, Amada Batista-Mainegra, Mercedes González Fernández-Larrea

DDI: http://dx.dai.org//1.22458/caes.vlli2.3324

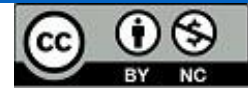

Artícula protegida par licencia Creative Cammans 
de la gestión de los procesos que tienen lugar en las IES, como una vía para elevar de forma progresiva su efectividad, y de la gestión institucional en general (Galarza y Almuiñas, 2010).

Los sistemas de gestión de la calidad (SGC) contribuyen de manera decisiva al logro de los objetivos de una organización, los cuales se adecuan a la institución en función de sus particularidades. Existen varias guías para organizar la confección de los SGC (Macías, 2010; Mira et al., 2010; Herrera y Arias, 2013; Ortiz; 2014; Normas ISO 9000, 9001, 9004, el modelo EFQM, entre otros). Al tener en cuenta las propuestas estudiadas y el ciclo de Deming (1989), se procedió a establecer un protocolo, a partir de elementos comunes y los intereses de la investigación, para el cual los autores de la presente investigación proponen siete etapas para elaborar un sistema de gestión de calidad (Figura 1). En cada una de las etapas se definió un grupo de acciones y se tuvo en cuenta los insumos y métodos a emplear para llevarlas a cabo (Tabla 1).

En la Figura 1 se presentan los componentes del SGC, el cual está sustentado en los siete principios de gestión de la calidad según las normas ISO 9001 (2015). En esta representación gráfica del SGC, las líneas continuas expresan relaciones de dependencia, las líneas discontinuas exhiben relaciones de retroalimentación, las flechas verticales indican relación de subordinación y las flechas horizontales indican relaciones de cooperación. La simbología empleada, permite manifestar la relación dialéctica y armónica entre todos los componentes de las siete etapas y entre estas, en función del cumplimiento del encargo social del proceso extensionista y la satisfacción de los usuarios, así como de las exigencias del entorno social, cultural, político, ambiental y económico.

Sistema de gestión de la calidad del procesa de extensión universitaria, una experiencia en la Universidad de La Habana.

Odette Ganzález-Apartela, Amada Batista-Mainegra, Mercedes González Fernández-Larrea

DDI: http://dx.dai.org//0.22458/caes.vlli2.3324

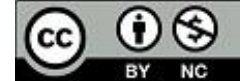

Artícula prategida par licencia Creative Cammans 


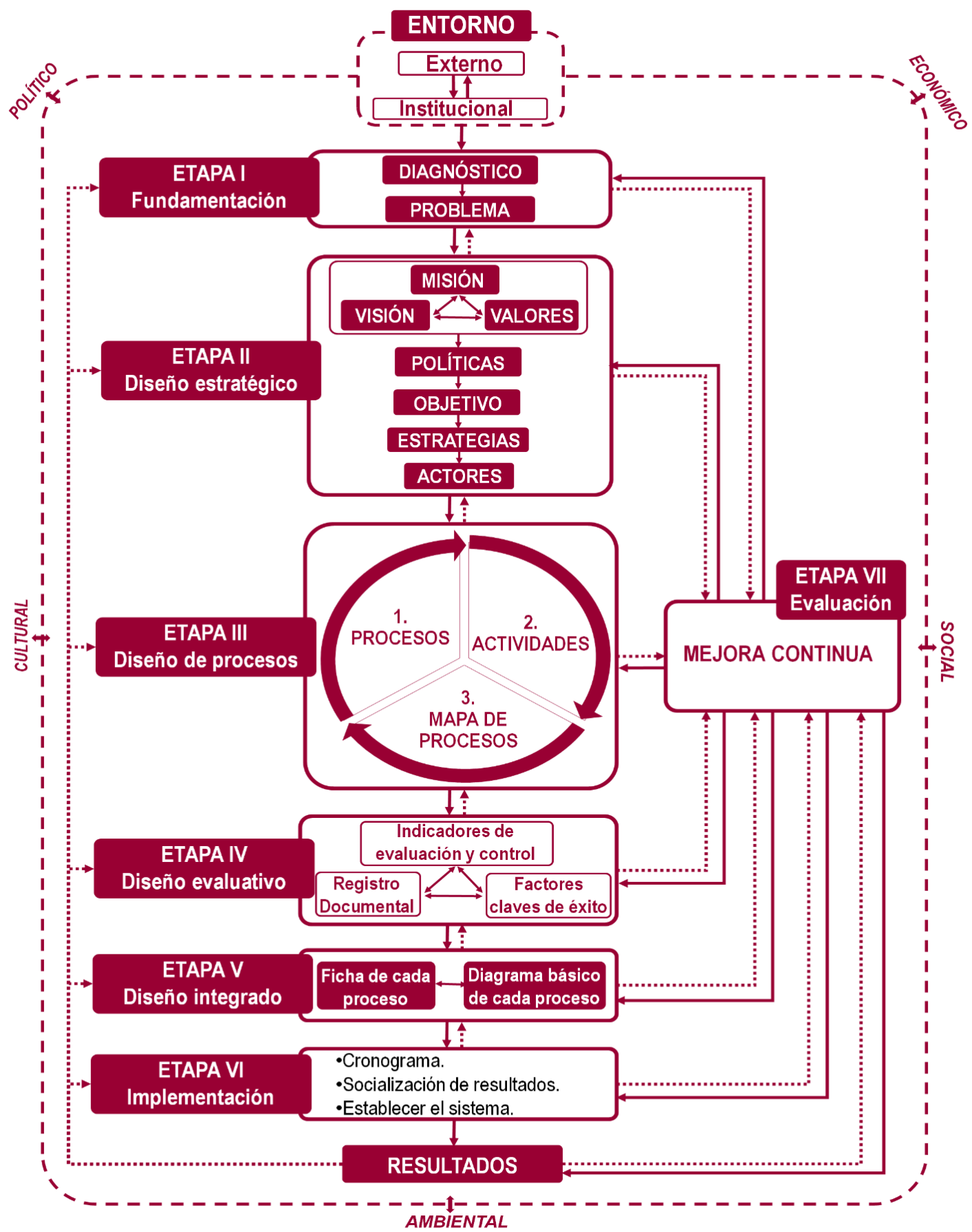

Figura 1. Diseño del sistema de gestión de la calidad. Fuente: González, 2016.

Sis tema de gestión de la calidad del procesa de extensión universitaria, una experiencia en la Universidad de La Habana. Odette González-Apartela, Amada Batista-Mainegra, Mercedes González Fernández-Larrea DII: http://dx.dai.org/l0.22458/caes.vlliZ.3324

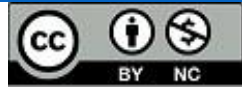

Artícula prategida par licencia Creative Cammans 
En el SGC expuesto están presentes los tres componentes básicos de un sistema de gestión definidos por Álvarez (2008), el componente sociopolítico, donde se identifican las políticas internacionales, nacionales, necesidades e intereses del entorno, que implican significados para la organización universitaria. El componente estratégico, en este se determinan las políticas institucionales y los objetivos, los cuales definen el horizonte de la organización, y se señalan los lineamientos que, con base en el componente sociopolítico y las políticas y objetivos institucionales, deben seguir quienes hacen efectivos los objetivos. Este componente está representado por la visión, la misión y la propuesta educativa de la institución en el caso de las universidades y el componente operativo, donde se identifican y desarrollan los procesos que hacen efectiva la estrategia y que están relacionados con el desarrollo del componente humano, los recursos físicos y financieros, los documentos y las operaciones que finalmente logran los objetivos.

Con base en el diseño del SGC propuesto y la relación de sus componentes y etapas, se determina que presenta las siguientes características generales:

- Abierto: permite la interacción constante y dinámica con el entorno institucional, político, económico, cultural, ambiental y social que posibilita, a través del seguimiento y retroalimentación continua, la toma de decisiones correctivas en el momento oportuno y de forma proactiva, en correspondencia con el principio de gestión de la calidad de toma de decisiones según los hechos.

- Flexible: puede ser aplicado a otros procesos universitarios de la UH y otras IES, adecuándolo en su implementación a las características propias de la institución, posibilita además el enriquecimiento de su propia concepción teórica a partir de la mejora continua del sistema.

- Contextualizado: porque responde a las necesidades y exigencias de entorno y la comunidad universitaria.

Sistema de gestión de la calidad del procesa de extensión universitaria, una experiencia en la Universidad de La Habana.

Odette Ganzález-Apartela, Amada Batista-Mainegra, Mercedes Gunzález Fernández-Larrea

DDI: http://dx.dai.arg/l0.22450/caes.vlli2.3324

(c) (i) \$)

Artícula prategida par licencia Creative Cammans 
- Carácter sistémico: relación dialéctica que se establece entre cada uno de sus componentes y etapas del SGC, lo que permite mejorar el proceso extensionista a partir de su transformación en la práctica, dada la posibilidad de evaluar y retroalimentar cada una de las acciones que se ejecutan a partir de la mejora continua.

Además, en el SGC propuesto se evidencian algunas de las dimensiones del proceso extensionista propuestas por Menéndez (2015), tales como Dimensión académicainvestigativa institucional, Dimensión social, cultural y productiva, Dimensión comunicacional, Dimensión política; así como una Dimensión tecnológica y otra económica, propuestas por Batista (2016).

Para llevar a cabo las diferentes etapas se desarrollan un conjunto de acciones, se tienen en cuenta el proceso y las IES en cuestión, tal y como se detalla en la Tabla 1.

Sistema de gestión de la calidad del procesa de extensión universitaria, una experiencia en la Universidad de La Habana. Odette Ganzález-Apartela, Amada Batista-Mainegra, Mercedes Gunzález Fernández-Larrea DII: http://dx.dai.org/10.22458/caes.vlliz.3324 
Carreco electrónica: revistacalidad国uned.ac.Сॅ

\begin{tabular}{|c|c|c|c|}
\hline Etapas & Acciones & Insumos & Métados \\
\hline I. Fundamentación & $\begin{array}{l}\text { - Efectuar el diagnóstico de la } \\
\text { situación real de la gestión de la } \\
\text { calidad de la extensión } \\
\text { universitaria. } \\
\text { - Determinar el problema o } \\
\text { contradicción fundamental a } \\
\text { resolver. }\end{array}$ & $\begin{array}{l}\text { - Infarme del trabajo de extensión universitaria en la } \\
\text { IES. } \\
\text { - Infarme del MES de cumplimiento de objetivas. } \\
\text { - Objetivas de trabajo del MES y la IES. } \\
\text { - Conacimienta del proceso extensionista en la IES par } \\
\text { parte de estudiantes, trabajadores dacentes, no } \\
\text { docentes y directivas. } \\
\text { - Demandas del entarna. }\end{array}$ & $\begin{array}{l}\text { - Revisión documental } \\
\text { - Cuestionario } \\
\text { - Entrevista } \\
\text { - Observación } \\
\text { - Triangulación } \\
\text { - Matriz DAFD }\end{array}$ \\
\hline \multirow[t]{2}{*}{ II. Diseño estratégico } & $\begin{array}{l}\text { - Elaborar la misión y la visión para la } \\
\text { gestión de la calidad del proceso de } \\
\text { extensión. } \\
\text { - Determinar los valores necesarios e } \\
\text { indispensables para el cumplimienta } \\
\text { de la misión y la visión. } \\
\text { - Elaborar los objetivas para el } \\
\text { cumplimienta de la misión y visión. }\end{array}$ & $\begin{array}{l}\text { - Diagnóstica elaborado. } \\
\text { - Misión y visión de la IES. } \\
\text { - Valores de la IES. } \\
\text { - Objetivas de trabaja del MES, la IES y el área. } \\
\text { - Drientaciones derivadas de los DACE. } \\
\text { - Lineamientas de la política económica y sacial del } \\
\text { país. } \\
\text { - Demandas del entorno. }\end{array}$ & $\begin{array}{l}\text { - Revisión dacumental } \\
\text { - Entrevista } \\
\text { - Dbservación }\end{array}$ \\
\hline & $\begin{array}{l}\text { - Definir la política y las estrategias } \\
\text { necesarias para cumplir la MVyV. } \\
\text { - Identificar los actares del procesa } \\
\text { (internos y externos) }\end{array}$ & $\begin{array}{l}\text { - Diagnóstica elabarado. } \\
\text { - Objetivas de trabaja del MES, la IES y el área. } \\
\text { - Orientaciones derivadas de los DACE. } \\
\text { - Lineamientas de la política ecanómica y sacial del } \\
\text { país. } \\
\text { - Demandas del entarno. }\end{array}$ & $\begin{array}{l}\text { - Revisión documental } \\
\text { - Entrevista } \\
\text { - Dbservación }\end{array}$ \\
\hline Ill.Diseño de procesos & $\begin{array}{l}\text { - Estructurar el macropracesa y los } \\
\text { procesos que lo integran. } \\
\text { - Diseñar el mapa de pracesos. } \\
\text { - Determinar las actividades } \\
\text { comprendidas en cada proceso. }\end{array}$ & $\begin{array}{l}\text { - Diagnóstico elaborado. } \\
\text { - MVyV de la IES. } \\
\text { - MVyV determinados en la etapa anterior. } \\
\text { - } \text { Dbjetivas de trabajo del MES, la IES y el área. } \\
\text { - Drientaciones derivadas de los DACE. } \\
\text { - Lineamientas de la política económica y social del } \\
\quad \text { país. } \\
\text { - Gustos y preferencias. Demandas del entorno y } \\
\text { necesidades sociaculturales }\end{array}$ & $\begin{array}{l}\text { - Revisión documental } \\
\text { - Entrevista } \\
\text { - Dbservación } \\
\text { - Cuestionario } \\
\text { - Triangulación }\end{array}$ \\
\hline IV. Diseño Evaluativo & $\begin{array}{l}\text { - Determinar los indicadores de } \\
\text { evaluación y control. } \\
\text { - Elaborar el registro de contral de } \\
\text { documentas para la evaluación y el } \\
\text { contral. } \\
\text { - Determinar los factares claves de } \\
\text { éxito. }\end{array}$ & $\begin{array}{l}\text { - Diagnóstica elabarada. } \\
\text { - MVyV de la IES. } \\
\text { - MVyV determinadas en la etapa II. } \\
\text { - Objetivas de trabajo del MES, la IES y el área. } \\
\text { - Orientaciones derivadas de los DACE. } \\
\text { - Lineamientas de la política ecanómica y sacial del } \\
\quad \text { país. }\end{array}$ & $\begin{array}{l}\text { - Revisión dacumental } \\
\text { - Entrevista } \\
\text { - Triangulación }\end{array}$ \\
\hline
\end{tabular}

Sis tema de gestión de la calidad del proceso de extensión universitaria, una experiencia en la Universidad de La Habana. Odette Gunzález-Apartela, Amada Batista-Mainegra, Mercedes Ganzález Fernández-Larrea DDI: http://dx.dai.org//0.22458/caes.vlli2.3324 


\begin{tabular}{|c|c|c|c|}
\hline & & - Demandas del entorno y necesidades sacioculturales. & \\
\hline V. Diseño integrado & $\begin{array}{l}\text { - Elaborar las fichas de cada proceso. } \\
\text { - Elaborar los diagramas básicos de } \\
\text { cada proceso. }\end{array}$ & $\begin{array}{l}\text { - Resultadas de las etapas anteriores. } \\
\text { - Modela de ficha y diagrama básico a emplear. }\end{array}$ & $\begin{array}{l}\text { - Revisión dacumental } \\
\text { - Triangulación } \\
\text { - IDEFD }\end{array}$ \\
\hline VI. Implementación & 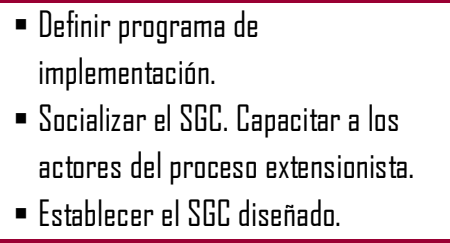 & $\begin{array}{l}\text { - Diagnóstica elaborado. } \\
\text { - Demandas del entorna y necesidades socioculturales. } \\
\text { - Cultura organizacional (sistema de trabajo del área). } \\
\text { - Condiciones de la IES/áreas universitarias. }\end{array}$ & $\begin{array}{l}\text { - Revisión dacumental. } \\
\text { - Dbservación } \\
\text { - Cuestionario }\end{array}$ \\
\hline VII. Evaluación & $\begin{array}{l}\text { - Ejecutar el contral operativo. } \\
\text { - Evaluar el sistema de indicadares } \\
\text { de gestión. }\end{array}$ & $\begin{array}{l}\text { - Planes de trabaja por área. } \\
\text { - Infarmes de las áreas universitarias. } \\
\text { - Dbjetivas de trabajo del MES, la IES y el área. } \\
\text { - Orientaciones derivadas de los DACE. }\end{array}$ & $\begin{array}{l}\text { - Revisión documental } \\
\text { - Dbservación } \\
\text { - Cuestionario. }\end{array}$ \\
\hline
\end{tabular}

Tabla 1. Desarrollo de las etapas del sistema de gestión de la calidad. Fuente: González, 2016.

Fuente: González, 2016.

\section{Etapa I. Fundamentación del sistema de gestión de calidad del proceso extensionista en la Universidad de La Habana}

En esta etapa se hace una caracterización de las IES y del proceso de extensión universitaria en el cual se toma en cuenta la universidad en su trayectoria histórica, política, cultural y social, la cual ha incorporado cambios que le han permitido mantener su vigencia como organización social en un mundo inmerso en transformaciones constantes. El proceso de extensión universitaria que permite de manera ágil y flexible interactuar con la sociedad su riqueza humanística, cultural y científica, su gestión eficiente contribuirá al cumplimiento de la misión de las IES. La gestión de la extensión universitaria es el motivo de esta investigación.

\section{Etapa II. Diseño estratégico del SGC de la extensión universitaria en la IES}

Si se parte de los referentes teórico-metodológicos asumidos, del diagnóstico de la gestión del proceso extensionista en la institución, de las concepciones de misión, visión y valores

Sistema de gestión de la calidad del procesa de extensín universitaria, una experiencia en la Universidad de La Habana.

Qdette Ganzález-Apartela, Amada Batista-Mainegra, Mercedes Ganzález Fernández-Larrea

DII: http://dx.dai.org/10.22458/caes.vlliz.3324

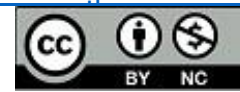

Artícula protegida par licencia Creative Commans 
que establece la red de educación superior de las IES (Domínguez, Galarza y Almuiñas, 2016), las definiciones de estos aspectos en el Ministerio de Educación Superior (MES) y la IES particular; así como el empleo de métodos teóricos y empíricos (entrevista grupal y cuestionarios) a profesores de la Dirección de Extensión Universitaria (DEU) y vicedecanos de las facultades que atienden el proceso, se determinaron por triangulación la misión, la visión y los valores del proceso extensionista en la $\mathrm{UH}$, en función de una gestión de calidad.

La misión determina lo que es esencial, la razón de ser o actividad particular de la organización, los fines últimos intermedios para los cuales fue creada y que otorgan sentido y valor a su existencia de actividad.

Los valores compartidos son las ideas fundamentales alrededor de las cuales se materializa la actividad de cualquier institución; "orientan conductas, cohesionan, dan sentido a las voluntades, estimulan el desarrollo, sirven como líneas directrices para la política, el pensamiento y el comportamiento de las personas dentro de la institución" (Domínguez, Galarza y Almuiñas, 2016, p. 23), por ello, el análisis y conceptualización de Ios valores en las IES debe desarrollarse en su nexo con la cultura organizacional.

Los valores compartidos orientan todas las acciones y decisiones que responden a un proceso de calidad de la extensión universitaria y definen los modos de actuación y compromiso para responder con calidad a todos los grupos de interés.

En coincidencia con Macías (2010), la misión, la visión y los valores (MVyV) de una organización, identifican el resultado esperado. La definición certera y transparente de estos elementos, permitirá la posibilidad real de materialización de la proyección del desarrollo institucional, como plantearan Galarza y Almuiñas (2015). Al tener en cuenta estos aspectos se prosigue con la determinación de la política y las estrategias.

Sistema de gestión de la calidad del procesa de extensión universitaria, una experiencia en la Universidad de La Habana.

Odette Ganzález-Apartela, Amada Batista-Mainegra, Mercedes González Fernández-Larrea

DDI: http://dx.dai.org/l0.22458/caes.vlliz.3324

(c)

Artícula prategida par licencia Creative Cammans 
Los autores del presente trabajo concuerdan con Macías (2010), al plantear que la política y la estrategia:

exponen las ideas que la organización pondrá en marcha con el fin de alcanzar los resultados esperados y definidos en la MVyV. La política y estrategia deben tener en cuenta los recursos disponibles y las condiciones del entorno en el que la organización desarrolla y desarrollará su actividad. (p. 16)

La política de la gestión del proceso extensionista, debe elaborarse en función del compromiso de guiar su gestión en consonancia con las políticas universitarias de la educación superior y en específico de las IES, así como, en función de elementos decisores de la política, la economía y la sociedad, además, debe contener la integración con los procesos de formación e investigación. Representa un "aspecto estratégico para implementar acciones colectivas eficaces que permitan asumir el protagonismo que le corresponde a la IES en la sociedad contemporánea, como institución que lidera el desarrollo cultural, científico, técnico y humanístico de la sociedad", concordando con Jaspe (2010, p. 104).

A partir de la política asumida, se define el objetivo del SGC y se parte de un análisis estratégico, según Lamolla (2014), el cual permite identificar en el entorno general, los factores en las diferentes esferas que influyen en el proceso. Estos factores son Políticos/Legal; Económico; Tecnológico; Sociocultural y medio ambiental (análisis PEST). Las estrategias por desarrollar estarán en conformidad con el análisis del entorno, la definición de la misión, la visión y los valores, que permitirán llegar al cumplimiento de las metas establecidas en función del enfoque de procesos. En este caso las estrategias a seguir serán: capacitación de los actores del proceso; implementación del sistema de gestión de la calidad del proceso extensionista en la UH con enfoque de gestión por

Sis tema de gestión de la calidad del procesa de extensión universitaria, una experiencia en la Universidad de La Habana.

Odette Ganzález-Apartela, Amada Batista-Mainegra, Mercedes González Fernández-Larrea

DDI: http://dx.dai.org//0.22458/caes.vlli2.3324

(c)

Artícula prategida par licencia Creative Cammans 
procesos; y retroalimentación del sistema de gestión diseñado para la UH en la medida que se capaciten e implementen el SGC.

Lograr la correcta asimilación y posterior cumplimiento de los aspectos valorados anteriormente solo será posible a partir de la participación activa de los actores. La responsabilidad que asuma cada actor es vital para el desarrollo de la gestión institucional, pues son estos los que diseñan y organizan los mecanismos y procedimientos, facilitan el flujo de información, coordinan el trabajo, distribuyen y ejecutan los recursos disponibles para el desarrollo de las actividades, por lo tanto, garantizan también el tránsito de la IES hacia niveles superiores de calidad. Es por ello, que capacitar a los actores en función de la gestión de la calidad y del proceso en particular que gestionan, posibilitará la necesaria comprensión y asimilación para el logro de los objetivos propuestos.

Lo cual está en concordancia con lo planteado por Jaspe (2010) al expresar que, la gestión de la extensión requiere de actores involucrados directa o indirectamente en el proceso de gestión de la extensión, que desplieguen acciones en forma consciente y organizada para operar y desarrollar los procesos extensionistas bajo determinados criterios de racionalidad y estén orientados a lograr resultados relevantes para la institución y la sociedad. (p. 113)

Domínguez, Galarza y Almuiñas (2016, p. 3) definen actores como "el conjunto de personas que trabajan unidos dentro de una institución de educación superior. La unión de varios actores da lugar a los colectivos de trabajadores involucrados en actividades o labores de algún tipo dentro de una IES". A partir de lo antes expuesto se determinan los actores del proceso extensionista en la IES. El proceso de extensión universitaria es una función sustantiva de la educación superior y por tanto de la IES, por ello sus actores son

Sistema de gestión de la calidad del procesa de extensión universitaria, una experiencia en la Universidad de La Habana.

Odette Ganzález-Apartela, Amada Batista-Mainegra, Mercedes González Fernández-Larrea

DDI: http://dx.dai.org/l0.22458/caes.vlliz.3324

(c)

Artícula prategida par licencia Creative Cammans 
todos los miembros de la comunidad universitaria (profesores, estudiantes, trabajadores y directivos) y los agentes culturales de la comunidad extrauniversitaria.

Los usuarios de las acciones del proceso extensionista de manera general, son todos aquellos que usan y perciben los resultados, así como el impacto del trabajo extensionista, estos se pueden dividir en internos y externos.

Los usuarios internos serían los estudiantes, profesores, investigadores, trabajadores en general, de la comunidad universitaria, y los externos: organismos de la administración central del Estado (OACE), las organizaciones políticas o de masas municipales, provinciales o nacionales, los miembros de las comunidades que interactúan con la universidad, las instituciones que desarrollan proyectos o acciones de promoción de la cultura con la institución, la sociedad en general.

Los actores del proceso pueden ser al mismo tiempo usuarios del proceso y los usuarios en ocasiones se pueden convertir en actores del proceso. Coincidiendo con González (2016),

un actor puede ser al mismo tiempo receptor de acciones de extensión de otra área universitaria, o de la misma área, pero que realiza acciones diferentes al sujeto en cuestión. Por ello, y se considera pertinente tener en cuenta este aspecto a la hora de la socialización de cada proceso en las diferentes áreas y espacios universitarios. (p. 89)

Una vez que se ha determinado y diseñado todos los aspectos estratégicos para la gestión del proceso extensionista, que determinan una gestión comprometida y coherente con las políticas nacionales y de la IES, se procede a diseñar los procesos y sus relaciones, las cuales harán efectiva la gestión.

Sistema de gestión de la calidad del procesa de extensión universitaria, una experiencia en la Universidad de La Habana.

Odette González-Apartela, Amada Batista-Mainegra, Mercedes González Fernández-Larrea

DDI: http://dx.dai.org//0.22458/caes.vlli2.3324

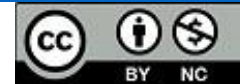

Artícula prategida par licencia Creative Cammans 


\section{Etapa III. Diseño de los procesos del SGC de la extensión universitaria en la} Universidad de La Habana

En vista de lo expresado anteriormente y en relación con las concepciones de gestión por procesos, el concepto de proceso de González (2016), y de las peculiaridades del proceso extensionista, se proponen los siguientes procesos a gestionar dentro del macroproceso de extensión universitaria en la Universidad de La Habana:

- Promoción de la cultura: Promueve cultura en su más amplia acepción, a partir de la realización de actividades y acciones, con la participación activa de estudiantes y profesores, para elevar la formación cultural de la comunidad universitaria y la sociedad.

- Formación y capacitación: Contribuye a la formación cultural integral de los integrantes de la comunidad universitaria y la sociedad, a través de la adquisición de conocimientos, habilidades y actitudes que favorezcan el desarrollo individual y colectivo.

- Gestión del movimiento de artistas aficionados: Desarrollo de aptitudes artísticas y de apreciación del arte, como parte de la formación integral de los jóvenes universitarios.

- Gestión del movimiento deportivo: Coordina y gestiona las actividades, competiciones internas y externas en sus instalaciones deportivas, puestas al servicio de la comunidad universitaria y su entorno, de tal forma que pueda conciliarse la vida académica y el deporte, estudio y ocio, que garantice una educación integral y de calidad.

- Gestión de proyectos: Desarrolla el trabajo sociocomunitario con la comunidad universitaria y la sociedad en interacción dialógica, en función de un problema social, a nivel comunitario, local, regional o nacional, potenciando la formación integral desde la perspectiva de la responsabilidad social universitaria.

Sistema de gestión de la calidad del procesa de extensión universitaria, una experiencia en la Universidad de La Habana.

Odette González-Apartela, Amada Batista-Mainegra, Mercedes González Fernández-Larrea

DDI: http://dx.dai.org//0.22458/caes.vlli2.3324

(c) (i) (5)

Artícula protegida par licencia Creative Cammans 
- Gestión de las instituciones culturales: Gestión integral de las instalaciones culturales, para promover cultura y prestar servicios, a disposición de la comunidad universitaria y la sociedad.

Los procesos se determinaron a partir de identificar actividades y tareas comunes, que los pudiesen constituir, teniendo en cuenta los fundamentos teóricos asumidos, coincidiendo con Álvarez (2008), el cual plantea determinadas características que deben cumplir los procesos, los cuales se expresan en los definidos anteriormente. Según este autor, para determinar si una actividad realizada por una organización es un proceso o subproceso, deben cumplir los siguientes criterios: la actividad tiene una misión o propósito claro, la actividad contiene entradas o salidas, se pueden identificar los usuarios, los proveedores y el resultado, servicio o producto final, la actividad debe ser susceptible de descomponerse en operaciones o tareas y la actividad puede ser estabilizada mediante la metodología de gestión por procesos (tiempo, recursos, costes). (Álvarez, 2008, p. 93)

Una vez definidos los procesos, se efectuaron entrevistas grupales con profesores de la DEU, vicedecanos de facultades y jefes de departamentos docentes, para retroalimentar y validar la propuesta. Los procesos definidos no poseen un orden jerárquico (Figura 2), su gestión depende de las entradas del proceso, de las necesidades y expectativas de las comunidades extra e intrauniversitaria, del entorno político, económico, ambiental, cultural y social y cada uno de ellos cumple un rol importante para lograr los resultados esperados por la organización.

Sistema de gestión de la calidad del procesa de extensión universitaria, una experiencia en la Universidad de La Habana.

Odette Gunzález-Apartela, Amada Batista-Mainegra, Mercedes Ganzález Fernández-Larrea

DDI: http://dx.dai.org/l0.22458/caes.vlliz.3324

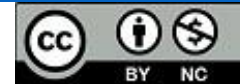

Artícula prategida par licencia Creative Cammans 


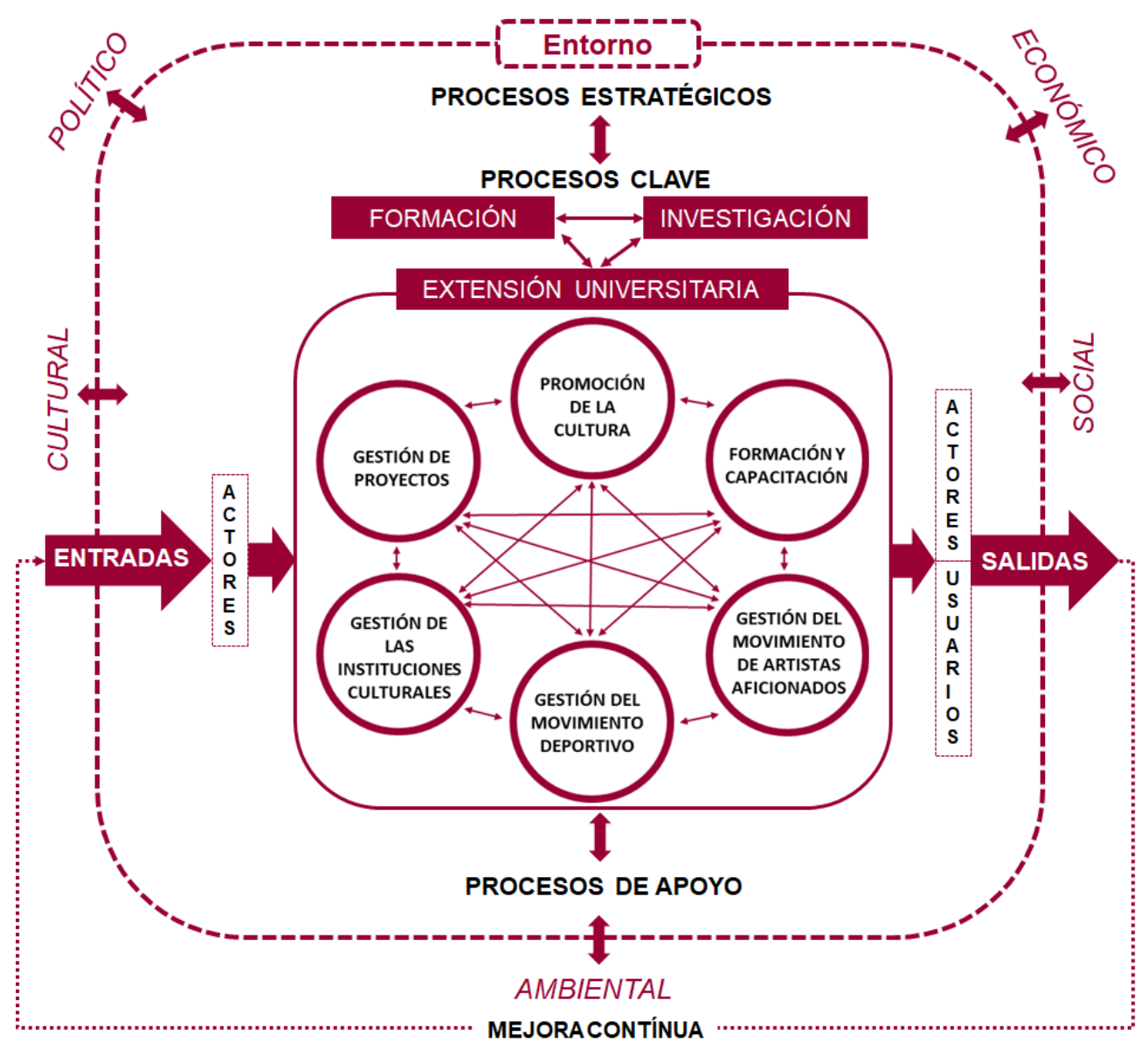

Figura 2. Mapa de procesos del macroproceso de extensión universitaria en la Universidad de La Habana desde un enfoque de gestión de la calidad.

Fuente: González (2016).

Según la literatura consultada, los procesos tradicionalmente identificados para gestionar la extensión universitaria son los del ciclo de gestión (organización, planificación, ejecución y control), los cuales son necesarios tenerlos en cuenta; sin embargo, los autores del presente trabajo consideran que estos son la base para gestionar cualquier proceso, pero no orientan de manera específica la gestión del proceso extensionista, por lo cual es

Sistema de gestión de la calidad del procesa de extensión universitaria, una experiencia en la Universidad de La Habana.

Odette Gunzález-Apartela, Amada Batista-Mainegra, Mercedes Ganzález Fernández-Larrea

DQI: http://dx.dai.org/10.22458/caes.vlliz.3324

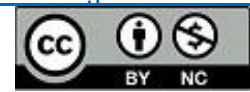

Artícula prategida par licencia Creative Cammans 
necesario ajustar los procesos de forma tal que pudiese servir de guía y favorecer la operacionalización, en la gestión de la extensión universitaria en cada área.

Los procesos de EU se interrelacionan entre sí y con los restantes procesos universitarios lo cual se observa en la Figura 2, donde se detalla el mapa de procesos que proporciona una perspectiva local-global y obliga a posicionar cada proceso respecto a la cadena de valor. Al mismo tiempo relaciona el propósito de la organización con los procesos que lo gestionan, utilizándose también como herramienta de consenso y aprendizaje.

En la Figura 2 se detalla cómo los procesos universitarios se interrelacionan unos con otros, pues los procesos claves dependen de los estratégicos y de los de apoyo; así como estos últimos se retroalimentan de los procesos clave para perfeccionar su gestión. La universidad en su totalidad depende del entorno, de los cambios y perspectivas de las condiciones sociales, políticas, culturales y económicas para llevar a cabo la gestión institucional.

Como se ha podido apreciar los procesos poseen relación entre ellos, manifestándose el funcionamiento del macroproceso como un sistema, donde cada uno de sus elementos están interconectados y retroalimentan a los otros, además interactúan con el resto de los procesos universitarios y el entorno.

Elaborar el despliegue de los procesos de la universidad implica una descripción detallada, dividiéndolos en subprocesos, actividades, tareas y en acciones, en dependencia del nivel de detalle o complejidad del proceso analizado. Para determinar las actividades por proceso es necesario tener en cuenta las entradas y los proveedores, luego se determinan los procedimientos y las salidas. Las actividades se determinan para cada proceso y poseen algunos procedimientos específicos, pero al mismo tiempo existen pasos comunes

Sistema de gestión de la calidad del procesa de extensión universitaria, una experiencia en la Universidad de La Habana.

Odette Ganzález-Apartela, Amada Batista-Mainegra, Mercedes González Fernández-Larrea

DDI: http://dx.dai.org/l0.22458/caes.vlliz.3324

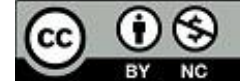

Artícula prategida par licencia Creative Cammans 
para su diseño y ejecución, para lo cual es indispensable tomar en consideración las condiciones del entorno, las relaciones con los otros procesos sustantivos, así como con los procesos de apoyo y estratégicos de la universidad, demostrando el carácter abierto, flexible y contextualizado del SGC.

\section{Etapas IV y V. Diseño evaluativo y diseño integrado del SGC del proceso extensionista de la Universidad de La Habana}

Una vez determinadas las actividades se procede a definir indicadores que contribuyen al éxito del sistema. Estos indicadores de evaluación permiten efectuar una adecuada medición, la cual requiere ser pertinente, significa que las mediciones llevadas a cabo deberán ser relevantes y útiles para facilitar las decisiones por tomar sobre la base de sus resultados; precisa, debe reflejar fielmente el comportamiento de las variables de medición, en este punto interviene la adecuada elección del instrumento de medición; oportuna, que los resultados de la medición estén disponibles en el tiempo en que la información es importante y relevante para la toma de decisiones, tanto para corregir como para prevenir; y económica, debe existir una proporcionalidad y racionalidad entre los costos incurridos en la medición y los beneficios o la relevancia de la información suministrada (Rodríguez et al., 2012).

En correspondencia con los indicadores de evaluación, es esencial para la IES saber qué espera la sociedad de ella, por lo que se deberán identificar los factores clave de éxito (FCE), estos pueden constituir fuerzas facilitadoras o barreras para la satisfacción de sus demandas. Las personas, los grupos humanos y las organizaciones que abordan un proyecto están interesados en conocer si se alcanza o no el éxito (Macías, 2010).

Se concuerda con lo planteado por Rodríguez et al. (2012) y Macías (2010) para proponer indicadores y factores que conduzcan al éxito del proceso, se tiene en cuenta los objetivos

Sistema de gestión de la calidad del procesa de extensión universitaria, una experiencia en la Universidad de La Habana.

Odette Ganzález-Apartela, Amada Batista-Mainegra, Mercedes González Fernández-Larrea

DDI: http://dx.dai.org/l0.22458/caes.vlli2.3324

(c) $(1)$ (S)

Artícula prategida par licencia Creative Cammans 
del MES y de la IES, así como su misión, además de los objetivos del proceso extensionista, por ello se proponen cuatro aspectos o indicadores fundamentales, a partir de los cuales podremos afirmar, si el proceso de extensión universitaria, en este caso de la UH, ha tenido éxito en su gestión.

Se deben especificar métodos de evaluación y revisión que constantemente permitan mejorar el proceso a partir de indicadores de calidad, por ello, se determinan los indicadores para cada uno de los procesos del macroproceso extensión universitaria según las variables a evaluar, las cuales se ubican en las fichas del macroproceso y específicamente en las fichas de cada proceso teniendo en cuenta los aspectos mencionados anteriormente.

Cada proceso dependerá de las entradas de información y del entorno, de la situación, económico-política y social, cultural y ambiental en la cual se encuentre la institución y la nación, los actores y de los proveedores de esa información.

En cada ficha se tienen presentes los elementos que corresponden al ciclo de gestión: planificar, organizar, ejecutar y controlar el proceso, lo cual corrobora lo planteado anteriormente, respecto a que esta propuesta no entra en contradicción con el ciclo básico de gestión, sino que en esta investigación de gestión por procesos se ajustan los procesos a las actividades y tareas que desarrolla la organización y en particular el proceso extensionista.

Una vez obtenidas las fichas se procede a la elaboración de los diagramas básicos de los procesos. En estos se refleja la secuencia ordenada de las actividades que lo integran, a las cuales se les debe asignar el responsable de su ejecución. Estas características se muestran de manera gráfica en el diagrama básico del macroproceso extensión

Sistema de gestión de la calidad del procesa de extensión universitaria, una experiencia en la Universidad de La Habana.

Odette Ganzález-Apartela, Amada Batista-Mainegra, Mercedes González Fernández-Larrea

DDI: http://dx.dai.org//0.22458/caes.vlli2.3324

(c) (i) (-)

Artícula prategida par licencia Creative Cammans 
universitaria, de igual manera se procede a elaborar los diagramas básicos de cada uno de los procesos determinados en el SGC. Una vez que se aplican las fichas y los diagramas básicos para la gestión del proceso extensionista, estos deben ir acompañados de una acción de mejora constante, la cual es toda acción destinada a cambiar la forma en que se está desarrollando un proceso. Estas se deben reflejar en una mejora de los indicadores del proceso, mediante aportaciones creativas, imaginación y sentido crítico.

\section{Etapas VI y VII. Implementación del SGC del proceso de extensión universitaria de la Universidad de La Habana}

La gestión por procesos concentra la atención en el resultado de cada uno de los procesos que desarrolla la institución, en vez de centrarlo en tareas o actividades. Cada persona que interviene en el proceso lo hace con la referencia del resultado final de la operación, en el caso de las IES, la atención se centra en hacer realidad la misión. Una institución es un sistema de sistemas, por lo tanto ningún director de área es el único responsable del buen fin de un proceso, pues la responsabilidad está repartida por áreas y en un mismo proceso intervienen varias áreas, por lo tanto la dirección general es la vía para responsabilizarse con todos y cada uno de los procesos, para identificar, entender y gestionar los procesos interrelacionados como un sistema, contribuyendo a la eficacia y eficiencia de la organización en el logro de sus objetivos, estos aspectos fueron tomados en cuenta como premisa para el diseño del SGC y su implementación.

Para la implementación del sistema de gestión diseñado, se siguieron los siguientes pasos: 1) Definir del programa de implementación del SGC; 2) Socializar los resultados del diseño del SGC; 3) Establecer el SGC y el Control operativo.

Sistema de gestión de la calidad del procesa de extensión universitaria, una experiencia en la Universidad de La Habana.

Odette Ganzález-Apartela, Amada Batista-Mainegra, Mercedes González Fernández-Larrea

DDI: http://dx.dai.org//0.22458/caes.vlli2.3324

(c) (i) (8)

Artícula prategida par licencia Creative Cammans 


\section{Evaluación del sistema de indicadores de gestión}

Se evaluó la satisfacción de los usuarios del SGC implementado al aplicar la técnica IADOV, la cual se ha convertido en un instrumento recurrente para la valoración de estrategias, metodologías y modelos en el área de las ciencias de la educación. Algunos investigadores, como Campistrous y Rizo (2006), recomiendan su empleo, pues al convertir a los usuarios de la propuesta en evaluadores de esta, los criterios emitidos al valorar los resultados resultan más contextualizados.

Una herramienta útil para definir, implementar y controlar las acciones correctivas y las mejoras es la metodología PHVA (Planificar-Hacer-Verificar-Actuar). EI PHVA (Deming, 1989) es una metodología dinámica que puede ser desplegada dentro de cada uno de los procesos de la organización y sus interacciones. Está íntimamente asociado con la planificación, implementación, verificación y mejora. Se puede lograr el mantenimiento y la mejora del desempeño del proceso mediante la aplicación del concepto PHVA en todos los niveles dentro de una organización. Esto se aplica igualmente a procesos estratégicos de alto nivel y a las actividades de operaciones sencillas.

El desarrollo de las acciones de mejora producto de los resultados de la evaluación desarrollada, permitió reajustar poco a poco los procesos que conforman el macroproceso de extensión universitaria, para obtener los resultados esperados. Entre las mejoras efectuadas se incluye, la simplificación del proceso, el aumento de la eficiencia, la mejora de la eficacia y la reducción del tiempo de ciclo del proceso. Al mismo tiempo es importante verificar la eficacia de las mejoras introducidas (ISO 9000, 2000).

El $100 \%$ de los encuestados manifestó conocer el SGC implementado, resaltando que el 95,2 \% mostró satisfacción con dicho sistema, al declarar que era muy efectivo o más efectivo que sistemas de gestión anteriores. Estos resultados permitieron determinar un

Sis tema de gestión de la calidad del procesa de extensión universitaria, una experiencia en la Universidad de La Habana.

Odette Ganzález-Apartela, Amada Batista-Mainegra, Mercedes González Fernández-Larrea

DDI: http://dx.dai.org/l0.22458/caes.vlliz.3324

(c)

Artícula prategida par licencia Creative Cammans 
Índice de Satisfacción Grupal (ISG) de 0,64, lo cual evidencia que en la muestra seleccionada existe satisfacción y reconocimiento de la efectividad y utilidad del SGC implementado durante cuatro cursos académicos.

La aplicación de la encuesta también permitió determinar que el $100 \%$ de los usuarios considera que el SGC implementado se caracteriza por ser contextualizado, en tanto el $90,5 \%$ expresó que dicho sistema es flexible y democrático, y el 85,7\% manifestó que era entendible y operativo.

Entre las causas de los diferentes niveles de satisfacción, específicamente las relativas al SGC implementado, destacan:

- el enfoque integrador y holístico al propiciar la integración con los procesos sustantivos de formación e investigación;

- la posibilidad de distinguir cada uno de los procesos que se incluyen en la extensión universitaria y la coherencia entre cada uno de estos, y

- la posibilidad real de desarrollar una medición integral del proceso, con indicadores cualitativos y cuantitativos, con una mirada enfocada hacia la calidad.

Con los elementos expuestos, referidos al SGC de extensión universitaria en la UH y con los resultados de la aplicación de la técnica IADOV, se pudo determinar que el sistema propuesto cumple con los principios de gestión de la calidad comprendidos en las normas ISO 9001 (2015).

\section{Conclusiones}

El sistema de gestión de la calidad del proceso de extensión universitaria en la Universidad de La Habana, garantiza su aplicación desde un enfoque de gestión por procesos,

Sistema de gestión de la calidad del procesa de extensión universitaria, una experiencia en la Universidad de La Habana.

Odette Gunzález-Apartela, Amada Batista-Mainegra, Mercedes Ganzález Fernández-Larrea

DII: http://dx.dai.org/l0.22458/caes.vlli2.3324

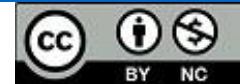

Artícula prategida par licencia Creative Cammans 
$\overline{\text { mediante una sucesión de siete etapas interrelacionadas, que parte de un diagnóstico }}$ inicial de las demandas del contexto externo e interno. Se caracteriza por ser abierto, flexible, contextualizado y tener un carácter sistémico, y se manifiestan las principales dimensiones de la extensión universitaria, así como una dimensión tecnológica y una económica.

Constituye una etapa vital en la implementación del sistema de gestión de la calidad propuesto, la capacitación de los diferentes actores, principalmente directivos del proceso en las facultades (vicedecanos, jefes y segundos jefes de departamento). La retroalimentación prevista en el SGC, permitió el control y la mejora continua de la implementación del sistema, a través de las diferentes actividades realizadas (visitas a las áreas universitarias, despachos con directivos del proceso, análisis de informes del trabajo extensionista y la observación de actividades) en los cursos en los cuales se evaluó el SGC.

La aplicación de la técnica IADOV permitió determinar que existe satisfacción y reconocimiento de la efectividad y utilidad del SGC implementado al obtener un Índice de Satisfacción Grupal de 0,64. Al mismo tiempo el 85,7\% de los usuarios, como mínimo, manifestó que el SGC se caracteriza por ser contextualizado, flexible, democrático, entendible y operativo.

Disponer de un Sistema de Gestión de la Calidad para el proceso de extensión universitaria, posibilita un mejor entendimiento de sus actores del proceso, a partir de la operacionalización de su gestión, lo que contribuye al logro de la misión institucional.

Sistema de gestión de la calidad del procesa de extensión universitaria, una experiencia en la Universidad de La Habana.

Odette González-Apartela, Amada Batista-Mainegra, Mercedes González Fernández-Larrea

DDI: http://dx.dai.org/l0.22458/caes.vlliz.3324

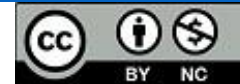

Artícula prategida par licencia Creative Cammans 


\section{Referencias}

Álvarez, M.G. (2008). Tesis doctoral "La gestión por procesos en la investigación universitaria, como búsqueda de calidad educativa. Propuesta de un modelo innovador de la gestión por procesos en la investigación. El caso de la pontificia universidad javeriana. Bogotá-Colombia". Universidad de Deusto. Bilbao, 367p.

Batista, A., Ortiz, T. y González, O. (2018). Integración de procesos sustantivos universitarios: contribución de la extensión universitaria a la promoción de salud. Revista Congreso Universidad, Vol. 7, No. 5, pp. 1-16.

CRES (2018). Declaración de la III Conferencia Regional de la Educación Superior para América Latina y el Caribe, Córdoba, Argentina.

Domínguez, J. J., Galarza, J. y Almuiñas, J. L. (2016). Glosario de términos vinculados a la Dirección estratégica en la Educación Superior. Universidad Regional Autónoma de los Andes, Ambato, Ecuador, 272p.

González, G. y González, M. (2020). Extensión universitaria y responsabilidad social de la universidad: Retos ante la agenda 2030. XV Taller Internacional de Extensión Universitaria. 12mo Congreso Internacional de Educación Superior, La Habana, Cuba. Memorias del evento, Editorial Universitaria, La Habana. ISBN 978-959-164408-4.

González, O. y Batista, A. (2019). Contribución de los proyectos integrados de Extensión Universitaria a la formación integral del futuro profesional. En: Formação docente e trabalho pedagógico: contextos atuais. Editora SCOTTI, GOIÃNIA, Brasil, pp. 55-70.

González, O. (2016). Sistema de gestión de calidad del proceso extensionista en la Universidad de La Habana. Tesis en opción al grado de Doctor en Ciencias de la Educación. CEPES, Universidad de La Habana, Cuba, 222p.

Jaspe, A. (2010). La gestión de la extensión universitaria y el compromiso social de la universidad. Rev. Informe de investigación educativa. Vol. XXIV, pp. 101-114.

Lemaitre, M., J., Aguilera, R., Dibbern, A., Hayte, C., Muga, A. y Téllez, J. (2018). La Educación Superior como parte del sistema educativo de América Latina y el Caribe. Calidad y Aseguramiento de la calidad. CRES. Universidad Nacional de Córdoba, 200p.

Sistema de gestión de la calidad del procesa de extensión universitaria, una experiencia en la Universidad de La Habana.

Odette González-Apartela, Amada Batista-Mainegra, Mercedes González Fernández-Larrea

DDl: http://dx.dai.org//0.22458/caes.vlli2.3324

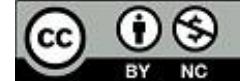

Artícula prategida par licencia Creative Cammans 
Macías, M. (2010). Dossier de trabajo. Resultados clave de un Servicio de Extensión Universitaria. Observatorio Cultural del Proyecto Atalaya, quinta fase. Universidad de Cádiz, España, 124p.

Maraña, M. (2010). Cultura y Desarrollo. Evolución y perspectivas. UNESCO Etxea, Cuadernos de trabajo. № 1, 30p. Disponible en: http://www.unescoetxea.org/dokumentuak/Cultura_desarrollo.pdf

MES (2010). Programa Nacional de Extensión Universitaria, actualización. Ministerio de Educación Superior, Cuba, p. 41.

Rodríguez, G., Lezcano, D., Varela, Y., Martínez, I. y Valdés, C. (2010). El enfoque de procesos como principio básico de los sistemas de gestión de calidad. Rev. Avances. Vol. 12, No. 1. CIGET, Pinar del Río, Cuba. 